\title{
NEFRECTOMÍA RADICAL LAPAROSCÓPICA EN ANGIOMIOLIPOMA RENAL CON INVASIÓN DE VENA RENAL*
}

\author{
Drs. Daniel Ruiz-Tagle P. ${ }^{1}$, Roberto Van Cauwelaert R. ${ }^{1}$, \\ Virginia Sanhueza L. ${ }^{2}$, Octavio A. Castillo C., ${ }^{1,3}$ \\ 1 Unidad de Urología, Clínica INDISA. \\ 2 Servicio de Anatomía Patológica, Clínica INDISA. \\ 3 Facultad de Medicina, Universidad Andrés Bello. \\ Santiago, Chile.
}

\begin{abstract}
\section{Laparoscopic radical nephrectomy of angiomyolipoma with renal vein involvement}

Background: Angiomyolipomas associated with tuberous sclerosis may invade the renal vein and generate intramural thrombi. Case report: We report a 36-years-old woman, consulting for left flank pain. CT scan showed a large tumor in the left kidney consistent with the diagnosis of infiltrating renal angiomyolipoma with tumor invasion of the vein. Laparoscopic nephrectomy was performed, with removal of tumor thrombus. The operative time was 127 minutes and estimated bleeding $20 \mathrm{ml}$. There were no intraoperative or postoperative complications. The patient is currently asymptomatic after 12 months of follow up. The pathological study of the surgical piece showed a renal angiomyolipoma with invasion of the kidney and a solid tumor in the lumen of the renal vein
\end{abstract}

Key words: Laparoscopic radical nephrectomy, angiomyolipoma, renal vein involvement.

\section{Resumen}

Objetivo: Se presenta el caso clínico de un Angiomiolipoma renal con extensión a vena renal, patología de muy baja frecuencia. Caso clínico: Mujer de 36 años, quien consulta por dolor en flanco izquierdo. En una tomografía computada se encuentra una lesión tumoral extensa del riñón izquierdo compatible con un Angiomiolipoma renal infiltrante e invasión tumoral de la vena renal. Se realiza nefrectomía laparoscópica, con extirpación de trombo tumoral. El tiempo operatorio fue de $127 \mathrm{~min}$ y el sangrado estimado de $20 \mathrm{ml}$. No hubo complicaciones intra ni postoperatorias. La paciente se encuentra actualmente asintomática luego de 12 meses de seguimiento. La histología mostró un Angiomiolipoma renal con invasión del riñón y un tumor sólido en el lumen de la vena renal. Conclusión: La invasión de vena renal por un Angiomiolipoma es extremadamente raro. Su resolución laparoscópica es posible, con sólo una comunicación previa en la literatura.

Palabras clave: Nefrectomía radical laparoscópica, angiomiolipoma, invasión de vena renal.

\footnotetext{
Los autores no refieren conflictos de interés.

Correspondencia: Dr. Octavio A. Castillo C. octavio.castillo@indisa.cl
}

*Recibido el 12 de abril de 2015 y aceptado para publicación el 2 de mayo de 2015. 


\section{Introducción}

El Angiomiolipoma es un tumor benigno del riñón, infrecuente $(0,3 \%-3 \%$ de los tumores renales), generalmente asintomático, de diagnóstico habitualmente incidental, que alcanza tamaños variables entre $2 \mathrm{~mm}$ a $20 \mathrm{~cm}$. Antes considerado un hamartoma, ahora es clasificado dentro de un grupo de tumores originados por la proliferación de células epitelioides perivasculares (PEComas), y está formado por tejido adiposo, vascular y muscular ${ }^{1}$. Es un tumor benigno, pero con crecimiento expansivo y riesgo de rotura espontánea cuando supera los $4 \mathrm{~cm}$ de diámetro. Tiene dos formas de presentación:

1. Una forma esporádica, más frecuente en mujeres (relación mujer/hombre de 4:1) y con una edad de presentación entre los 45 y 55 años.

2. En el síndrome de Esclerosis Tuberosa en donde su edad de presentación es entre los 25 y 35 años, pueden ser multifocales, y sin diferencias en cuanto al sexo $\mathrm{s}^{2}$. Se ha descrito la invasión del seno renal y formación de trombo tumoral en la vena, con ascenso de éste incluso hasta aurícula $^{3}$, de rara ocurrencia, siendo su resolución quirúrgica altamente compleja. Se han publicado aproximadamente 50 casos en la literatura de angiomiolipoma renal asociado a trombo tumoral venoso, y sólo uno caso manejado completamente por vía laparoscópica ${ }^{4}$. Describimos lo que creemos es el segundo angiomiolipoma tratado mediante nefrectomía y trombectomía por vía laparoscópica.

\section{Caso clínico}

Mujer de 36 años, previamente sana, quien consulta por historia de dolor en flanco izquierdo de varios meses de evolución. La tomografía computada describe una lesión tumoral compatible con un angiomiolipoma renal izquierdo de $6 \mathrm{~cm}$ de diámetro (Figura 1), con extensión a la vena renal principal (Figura 2). Se realiza nefrectomía laparoscópica, comprobando la presencia de trombo venoso con extensión hasta la vena cava, el que se desplaza hacia la vena renal y se controla mediante clips Hem-O-Lok®. El tiempo operatorio fue de 127 min y el sangrado estimado de $20 \mathrm{ml}$. Se da el alta a las $24 \mathrm{~h}$, sin incidentes. No hubo complicaciones intra ni postoperatorias. La paciente se encuentra actualmente asintomática luego de 12 meses de seguimiento.

La evaluación macroscópica muestra una lesión tumoral de superficie adiposa que ocupa polo inferior y tercio medio del riñón y que mide
$10,2 \times 4,2 \times 3,6 \mathrm{~cm}$. Se extiende a través de la cápsula renal y se continúa con el tejido adiposo perirenal. A nivel de la vena renal se aprecia trombo tumoral de aspecto adiposo de 2,5 × 2 × 1,2 cm, focalmente adherido a las paredes de la vena (Figura 3). La histología confirma se trata de un Angiomiolipoma renal, con moderada celularidad y leve pleomorfismo, con recuento mitótico de 0 mitosis en 10 campos.

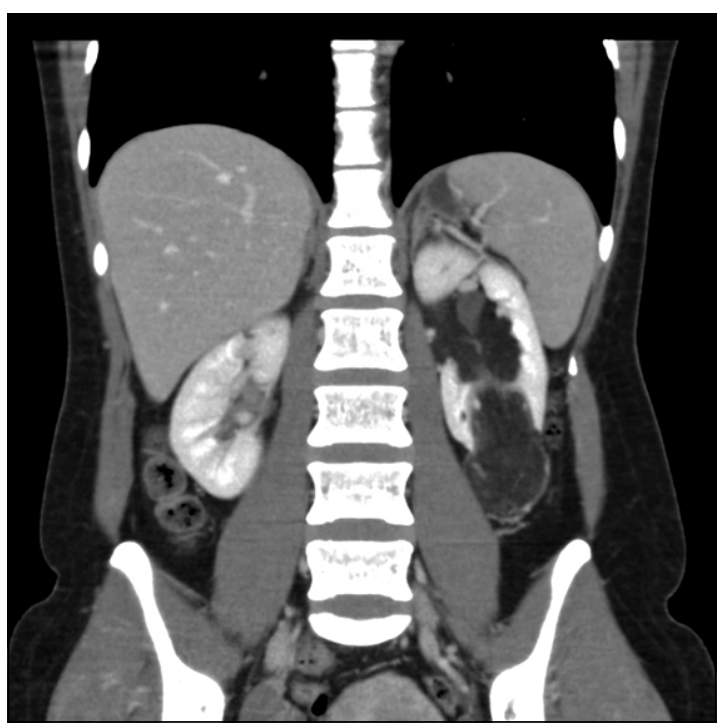

Figura 1. Tu renal.

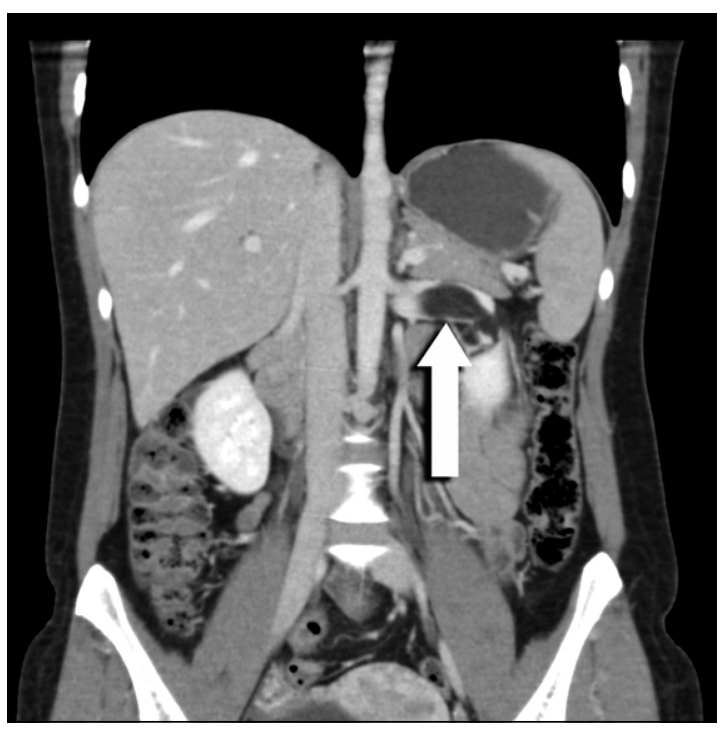

Figura 2. Extensión a vena renal. 


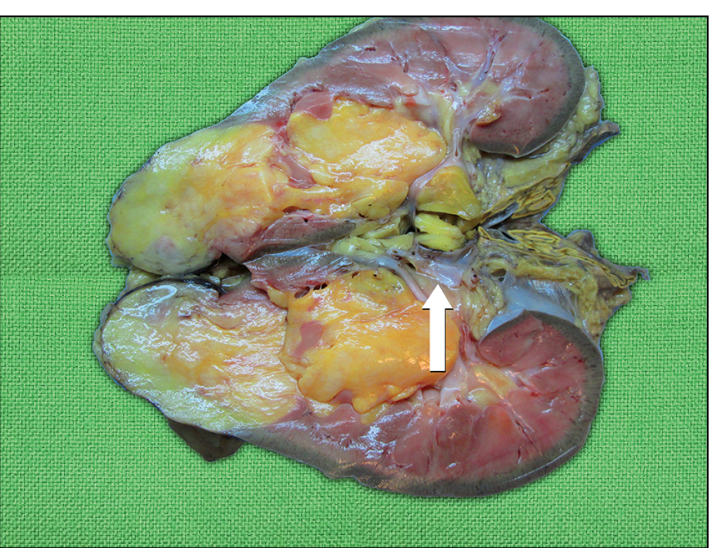

Figura 3. Trombo tumoral en vena renal.

\section{Discusión}

El interés clínico de los Angiomiolipomas es que puede ocurrir un rápido crecimiento, hay dificultad en el diagnóstico diferencial con lesiones malignas, siendo difícil establecer un diagnóstico seguro y un tratamiento correcto. Las lesiones mayores de $4 \mathrm{~cm}$ pueden causar dolor, ser palpables, e incluso presentarse como una emergencia médica dado que son la causa más frecuente de rotura renal espontánea ${ }^{5-8}$.

La indicación de tratamiento se hace en lesiones que crecen, se hacen sintomáticas o el diagnóstico es dudoso. El espectro va desde la nefrectomía parcial o radical, e incluso la embolización selectiva ${ }^{9,10}$.

El primer caso descrito de AML con trombo venoso fue descrito por Kutcher et al. ${ }^{11}$ en 1982 y desde entonces han sido publicados menos de 50 casos en la literatura mundial.

El manejo clásico del trombo tumoral en vena ha sido siempre a través de cirugía abierta, aunque en casos seleccionados podría realizarse por vía laparoscópica. En el caso descrito por FernándezPello y cols. ${ }^{4}$, realizan la nefrectomía en forma laparoscópica, pero utilizan pinza de Satinsky laparoscópica para la resección de trombo venoso que se extiende hasta vena cava. En nuestra experiencia $^{12}$, la cirugía laparoscópica es factible en pacientes con carcinoma de células renales y trombo venoso a ese nivel.

Describimos un raro caso de un tumor renal, habitualmente benigno, con extensión a la vena renal, de rara ocurrencia, tratado con cirugía de mínima invasión.

\section{Conclusiones}

La invasión de vena renal por un Angiomiolipoma es extremadamente rara. Su resolución laparoscópica es posible, con sólo una comunicación en la literatura.

\section{Referencias}

1. Bonetti F, Pea M, Martrigoni G, Zamboni G, Manfrin E, Clombari R, et al. The perivascular epithelioid cell related lesions. Adv Anat Pathol. 1997;4:343-58.

2. Eble JN, Sauter G, Epstein JI, Sesterhenn IA. Tumours of the Urinary System and Male Genital Organs. WHO Classification of Tumours; 67-70.

3. Davydov MI, Matveev VB, Lukianchenko AB, Kudashev BV, Petrovichev NN. Renal angiomyolipoma extending into the right atrium. Urol Int. 2001;67:168-9.

4. Fernández-Pello S, González Rodríguez I, Rodríguez Villamil L, Pérez-Carral JR, Luz Menéndez C, Caveda $\mathrm{P}$, et al. Laparoscopic management of right renal angiomyolipoma with involvement of the inferior vena cava: Case report and review of the literature. Scand J Urol. 2013;47:340-4.

5. Steiner MS, Goldman SM, Fishman EK, Marshall FF. The natural history of renal angiomyolipoma J Urol. 1993; 150:1782-86.

6. Molina Escudero R, Castillo OA. Spontaneous retroperitoneal hemorrhage of renal origin (Wunderlich syndrome): analysis of 8 cases. Arch Esp Urol. 2013;66:925-9.

7. Bolufer E, López-Fontana G, Castillo OA. Robot assisted partial nephrectomy (Da Vinci) in an angiomyolipoma associated to Wünderlich Syndrome. Arch Esp Urol. 2012;65:831-4.

8. Molina Escudero R, Castillo OA. Hemorragia retroperitoneal espontánea de origen renal (síndrome de Wunderlich): análisis de 8 casos. Arch. Esp. Urol. 2013;66:925-9.

9. Castillo OA, Fonerón A, Vidal-Mora I, Tapia C. Nefrectomía parcial laparoscópica en angiomiolipoma gigante. Rev Chil Cir. 2008;60:442-6.

10. Azócar G, Castillo O, Van Cauwelaert R, Aguirre C, Wöhler C, Wash A. Esclerosis tuberosa sangrante: Caso clínico en un paciente Testigo de Jehová. Rev Med Chile 1999; 127:1365-8.

11. Kutcher R, Rosenblatt R, Mitsudo SM, Goldman M, Kogan S. Renal angiomyolipoma with sonographic demonstration of extension into the inferior vena cava. Radiology 1982;143:755-6.

12. Castillo O, Silva A, Alemán E. Laparoscopic management of renal tumors with macroscopic level 1 renal vein involvement. Urol Int. 2015;94:88-92. 